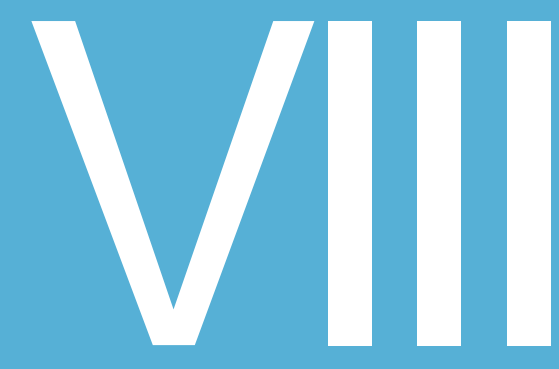

\title{
NIVEL DE MADUREZ DE LOS PROCESOS DE LA GESTIÓN DE SERVICIOS EN BASE A BPM
}

\section{Maturity level in Management Services Processes' based on BPM}

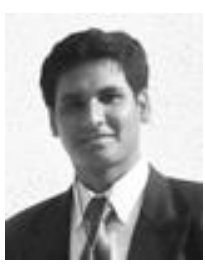

\section{Joseph Cruz}

Bachiller en Ingeniería de Sistemas por la Universidad Peruana Unión. Ponente e investigador visitante.

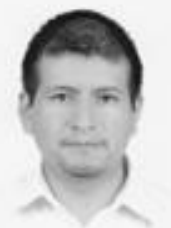

\section{Daniel Lévano}

Ingeniero de Sistemas por la Universidad Peruana Unión. Magíster en Ingeniería de Sistemas por la Universidad Peruana Unión. Docente Universitario de la Facultad de Ingeniería y Arquitectura y actual coordinador de Ingeniería de la Universidad Peruana Unión, sede Tarapoto. 


\section{Resumen}

Las empresas, a nivel global, tienden a una mayor dependencia de las Tecnologías de la Información ( $\mathrm{TI})$, no solo para el mantenimiento operativo de las instancias de la organización, sino también para el aumento de valor a la empresa por medio de la explotación de datos y sobre todo bajo el análisis y optimización de sus procesos. La metodología Business Process Management (BPM), al combinarlas con las buenas prácticas propuestas por Information Technology Infrastructure Library (ITIL), permite la posibilidad de aumentar el valor de la entidad por medio de la mejora y adaptación de los procesos desde una perspectiva más ágil, automatizada y robusta con la capacidad de adaptación al cambio, permitiendo a las organizaciones orientar sus procesos al cliente.

Palabras clave: Buenas prácticas, calidad, eficacia, gestión, implantación, BPM, información, ITIL, planeación, proceso, tecnología de información.

\section{Abstract}

Companies at global level, use to suffer a higher dependency on information technology, not just for operational maintenance of level of organization levels, but also for increasing the business worth through use of data and specially through analysis and optimizing its processes. The methodology Business Process Management (BPM) when combining with the good practices proposed by Information Technology Infrastructure Library (ITIL), it gets the opportunity to increase the value of any company through processes improvement and adjustment from a better and agile view, stronger with the ability to adapt to adjustments, allowing the organizations to aim their processes to the customer.

Keywords: Best practices, quality, efficiency, management, implementation, BPM, information, ITIL, planinng, process, information technology. 


\section{Introducción}

Actualmente son muy notorios los cambios que el mundo empresarial está experimentando, la búsqueda de nuevas formas de desarrollar los trabajos, conllevan a las organizaciones a buscar estándares y buenas prácticas, siempre certificado por terceras personas, que validen que lo que se está desarrollando esté correcto.

La aparición de ITIL marca un inicio de la estandarización en la administración de servicios desde una perspectiva del proceso del negocio. La notoriedad de ésta acrecienta a medida que la dependencia en la tecnología de la información aumenta, todo esto en búsqueda de utilizar con eficiencia los recursos materiales como no materiales.

Garimella y otros (2008), comentan que por otra parte BPM es un aglutinado de herramientas, métodos y tecnología utilizados para diseñar, representar, analizar y controlar procesos de negocio operacionales que a su vez presenta una visión orientada a procesos que ayudan a mejorar el desenvolvimiento de las operaciones combinando las TI.

Se pretende acentuar el nivel de desarrollo en la implantación de los procesos de la gestión de servicios, mostrando un alineamiento entre la optimización y las buenas prácticas propuestas por BPM e ITIL respectivamente.

\section{Ciclo de vida del proceso}

Carrasco (2006), menciona que teniendo presente los procesos de la gestión de servicios propuestos por ITIL en su tercera versión, se define el ciclo de vida del proceso BPM para su integración a las fases de implementación en ITIL y los niveles de madurez BPM.

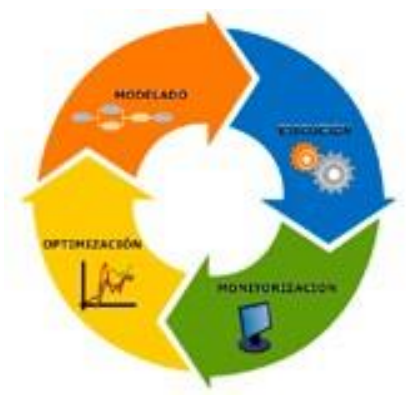

Figura 1 - Ciclo de vida del proceso (www.tcpsi.com 2011) 
Modelado. Describe el diseño organizacional, también la definición y limitaciones de roles y el flujos del desarrollo de trabajo.

Ejecución. Se aplica la ejecución de los procesos, se afirma la lista de tareas, alarmas y avisos, reglas de negocio, enrutamiento y asignación de tareas, gestión de documentos e integración.

Monitorización. Representa las métricas operacionales y de negocio definible, los cuadros de mando, como también el uso de recursos y la gestión de alertas y avisos.

Optimización. Implica la retroalimentación, análisis situacional, implementación de nuevos procesos y cargado a los de procesos modificados.

\section{Nivel de madurez BPM}

El nivel de madurez presenta la vista holística de una implementación BPM, basado en esta perspectiva, se puede definir alineamientos sólidos entre la implementación cooperativa de una administración basada en procesos bajo los patrones de buenas prácticas.

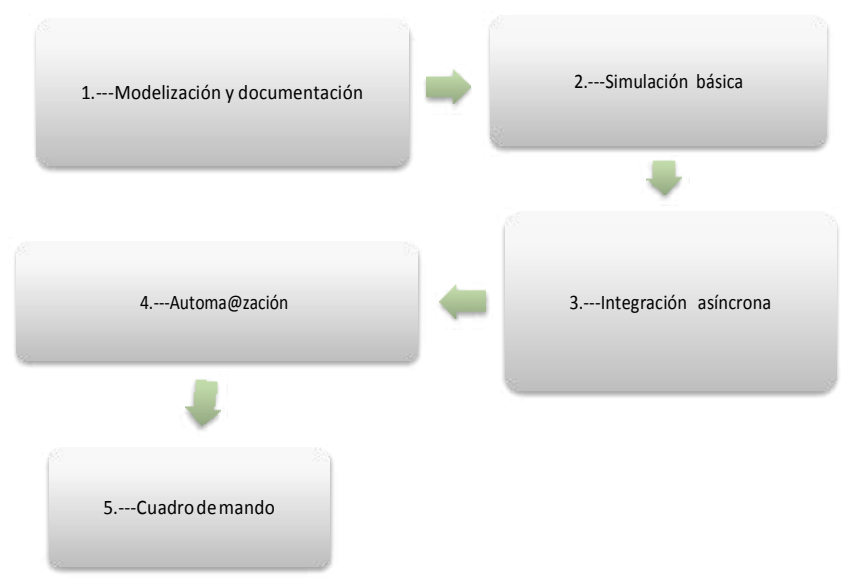

Figura 2 - Nivel de madurez BPM (Carrasco 2006) 


\section{Fases de la implementación de procesos de la gestión de servicios}

Ocampo (2009), describe que cada una de estas fases integra un flujo propio de trabajo, uno dependiente del otro (secuencial) indispensable para lograr el objetivo trazado de sus fases dependientes.

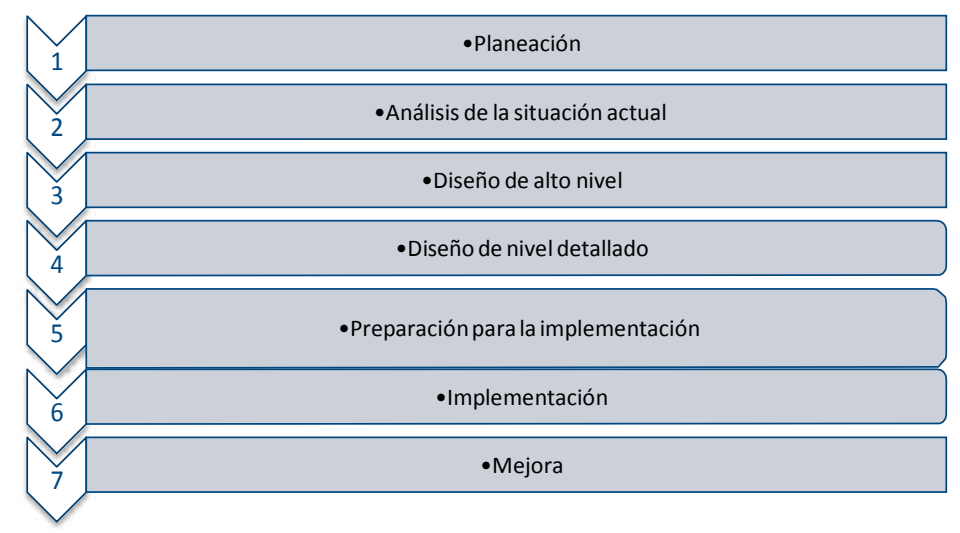

Figura 3 - Fases de la implementación de procesos de la gestión de servicios (Ocampo 2009)

\section{Requerimientos básicos para la implementación BPM}

Vásquez y otros (2010), definen los siguientes conceptos:

Definición de objetivos. Como primer requerimiento se tiene la definición del objetivo de la administración, con la premisa de alinear las estrategias de la entidad con el uso de BPM, todo esto para lograr un planteamiento claro con mira a la misión y visión de la organización.

Modelado. Seguido del primer requerimiento el modelado de los procesos con base al conocimiento y definición de cada una de ellas, con esto se pretende identificar aquellas actividades y tareas críticas que posiblemente pueden interferir en el resultado. 
Organización de equipo. Se determina la estructura del equipo de BPM que va a permitir organizar los perfiles para la implementación, siendo esto fundamental para su éxito de la madurez de la disciplina al interior de la empresa. Simulación. Después se realiza un trabajo de simulación del modelo definido (en la herramienta que mejor se adapte a nuestras necesidades y conocimiento), considerando cada una de las variables que posiblemente puedan interferir de forma positiva o negativa en los procesos de negocio.

Como agregado se recomienda utilizar una lista de chequeo y documentar el plan de implementación, para garantizar así un seguimiento apropiado de cada uno de los pasos a seguir dentro del proceso permitiendo conocer puntos críticos y requerimientos propios del equipo.

\section{Definición de las fases de la implementación de procesos de la gestión de servicios}

Ocampo (2009), define los siguientes:

Planificación. Se define la estrategia del proyecto, las actividades de planeación y logística necesarias para la ejecución de las etapas siguientes. En esta etapa es de vital importancia la comunicación de la apertura de la adaptación en la entidad, como también el establecimiento de objetivos y metas; y de las asociaciones de equipos de labor.

Análisis de situación actual. Se identifica la forma, cómo trabaja la organización considerando las funciones, roles, actividades y procesos. En esta etapa se mapean las estructuras jerárquicas/ funcionales y los problemas operativos actuales; con esto se identifican las oportunidades de mejora.

Diseño de alto nivel. Se determina el marco de referencia de mejores prácticas para el modelo a desarrollar. Se construye un modelo ideal considerando los principales procesos, con lo que se hace un análisis de la situación actual para diseñar el modelo de dirección futuro.

Diseño de nivel detallado. El nivel detallado debe proveer la información necesaria para la operación del modelo. Incluye instrucciones detalladas de procesos, identificación de roles, definición de formatos y selección de herramientas y definición de políticas aplicables. 
Preparación para la implementación. Para esta fase existe una serie de reglas y acti previas que se debe realizar antes de ejecutar la implementación, como la adquisición de herramientas, la realización de cambios organizacionales, cambios de los reglamentos internos, preparación de infraestructura.

Implementación. Se pone en marcha los nuevos procesos, tecnología de información y organización.

Mejora. Se establece mecanismos para asegurar la mejora continua a lo largo del tiempo y de esta manera identificar nuevas áreas de oportunidad. En esta fase son útiles las métricas recomendadas por alguna organización certificadora, por ejemplo el Organismo Internacional de Normalización o la Comisión Electrotécnica Internacional, como también se puede hacer uso de Service Level Agreement, entre un proveedor de servicios.

\section{Determinación de métricas y/o indicadores de evaluación}

Las métricas de evaluación pueden ser determinadas teniendo en consideración la evaluación del análisis de la situación actual con las expectativas de la misma, agregando datos obtenidos bajo la simulación de los procesos.

La evaluación de los parámetros deseados va en consecuencia a la flexibilidad de BPM y el análisis de la situación actual, permitiendo mantener la monitorización en la fase de implementación.

\section{Relación de implementación entre el Nivel de Madurez BPM y el Proceso de Gestión de Servicio}

Desarrollo de la implementación detallado basado en las caracterizas de proceso de implementación de ITIL y del nivel de madurez BPM.

\section{Primera relación}

En el desarrollo de los Procesos de Gestión de Servicios toda entidad u organización se encuentra primero en la planeación siendo parte de la modelización y documentación del nivel de madurez BPM. Esto implica 
el acceso a la documentación necesaria y su publicación para su análisis de la situación actual, teniendo como objeto delimitar el alcance del proyecto.

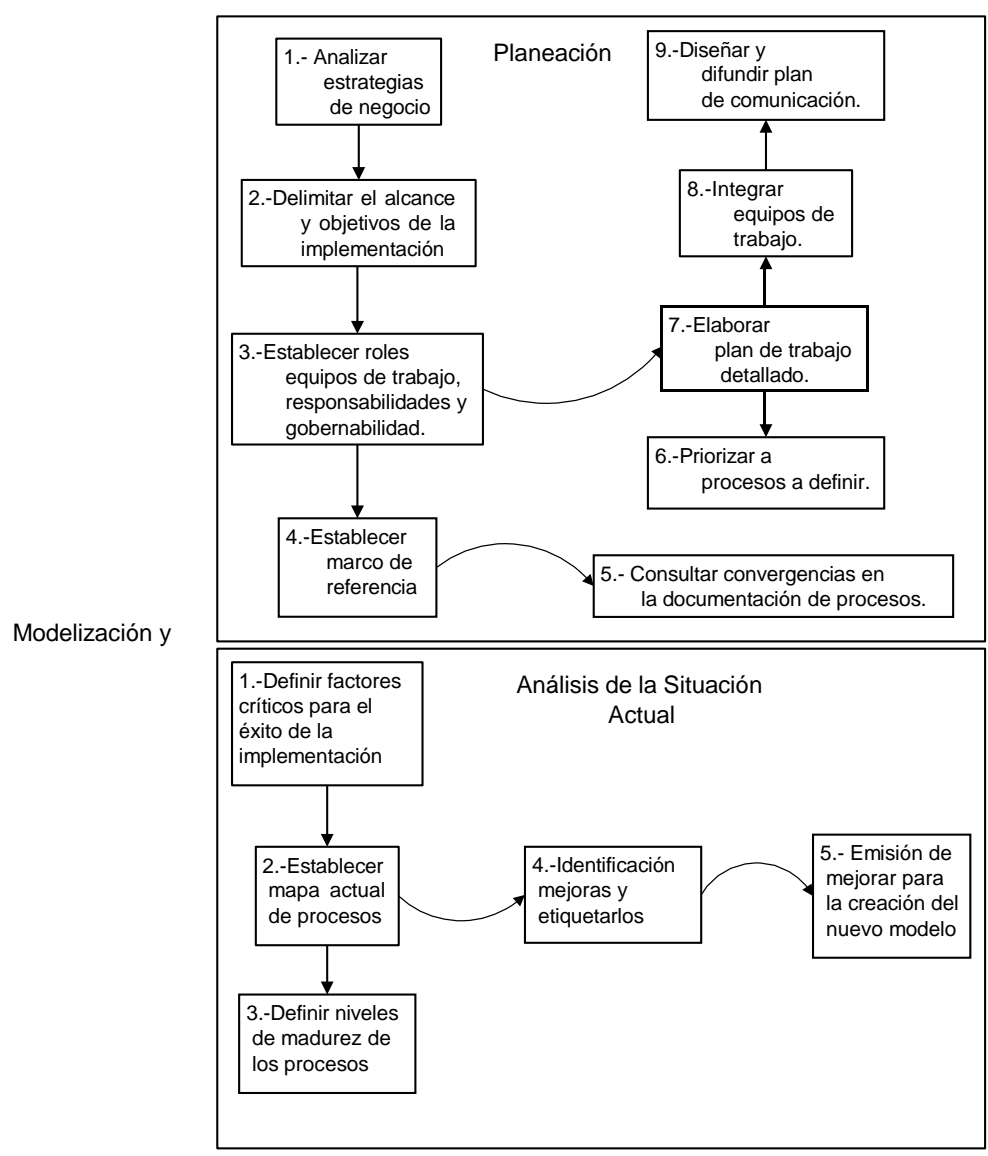

Figura 4 - Primera relación (Ocampo 2009)

\section{Segunda relación}

En esta relación la entidad ya se encuentra preparada para simular sus procesos con datos extraídos en la primera relación. 


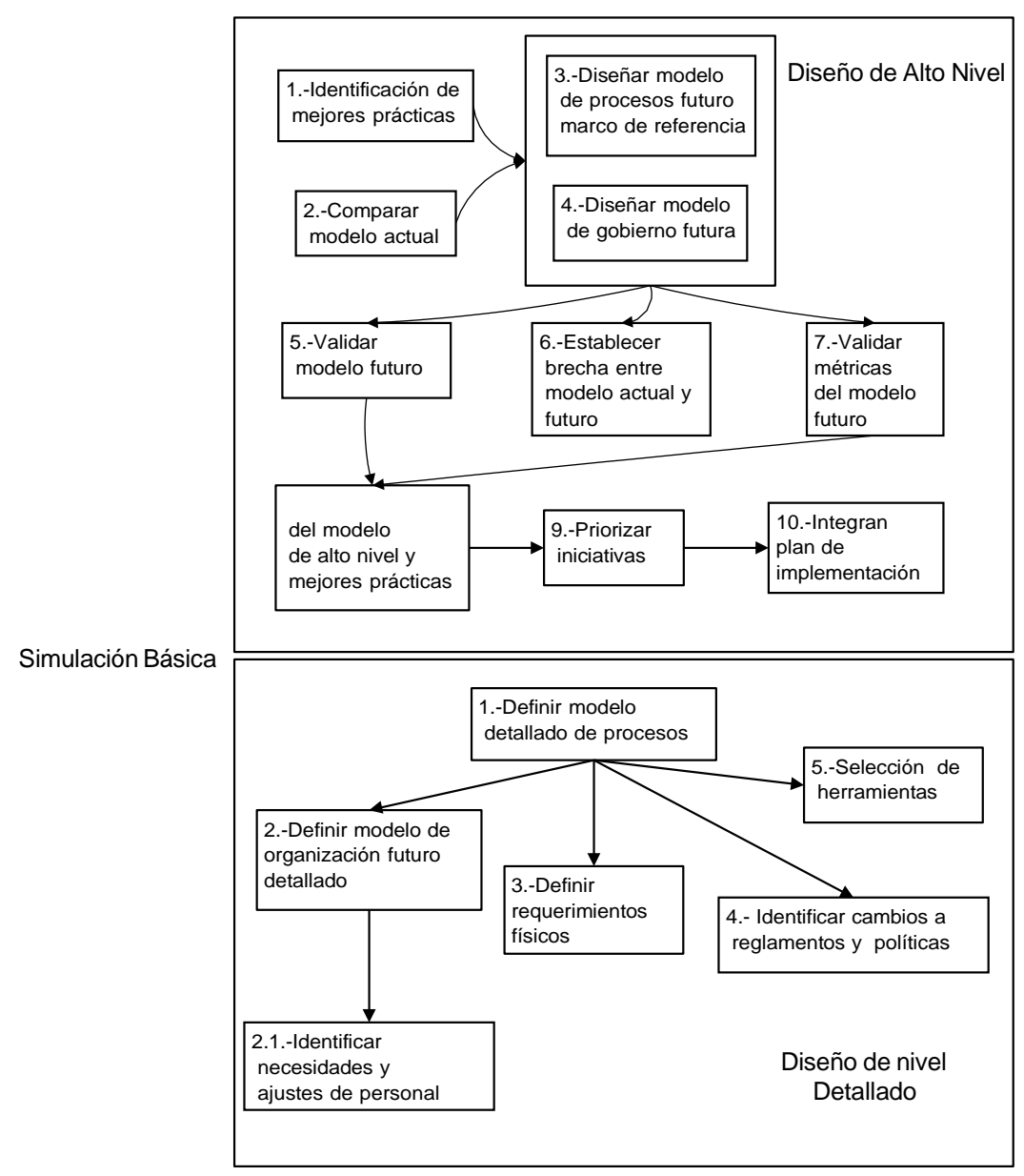

\section{Tercera relación}

Figura 5 - Segunda relación (Ocampo 2009)

La tercera relación comprende un lazo entre los niveles de madurez de integración asíncrona y la automatización, siendo la preparación para la implementación de los procesos de gestión de servicios la que equilibra esta brecha en el nivel de madurez permitiendo integrar los procesos, asignar las 
tareas, teniendo particular consistencia con la simulación ya obtenido previamente.

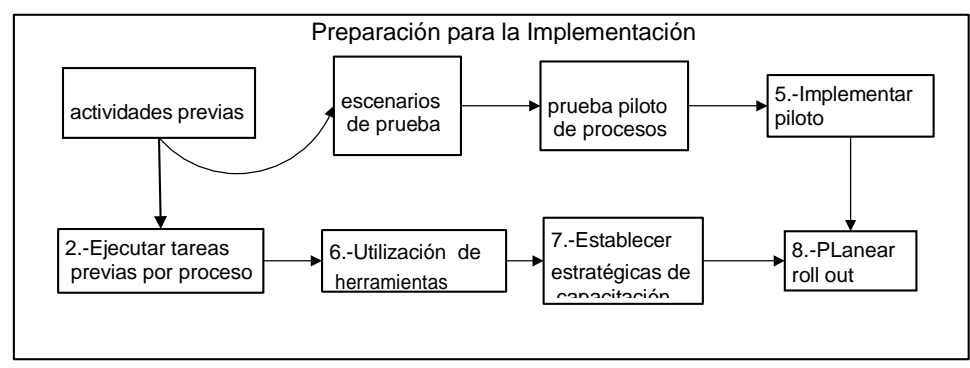

$$
\begin{aligned}
& \text { Integración } \\
& \text { asíncrona }
\end{aligned}
$$

Figura 5 - Tercera relación (Ocampo 2009)

\section{Cuarta relación}

Es la penúltima relación existente entre el nivel de madurez BPM y los procesos de gestión de servicios, aquí al igual que la tercera relación existe una correspondencia entre la automatización y el cuadro de mando, preparándose para la monitorización realizando pruebas de implementación de los procesos en tiempo real como también se realiza la revisión de las métricas que en el cuadro de mando se van a mostrar.

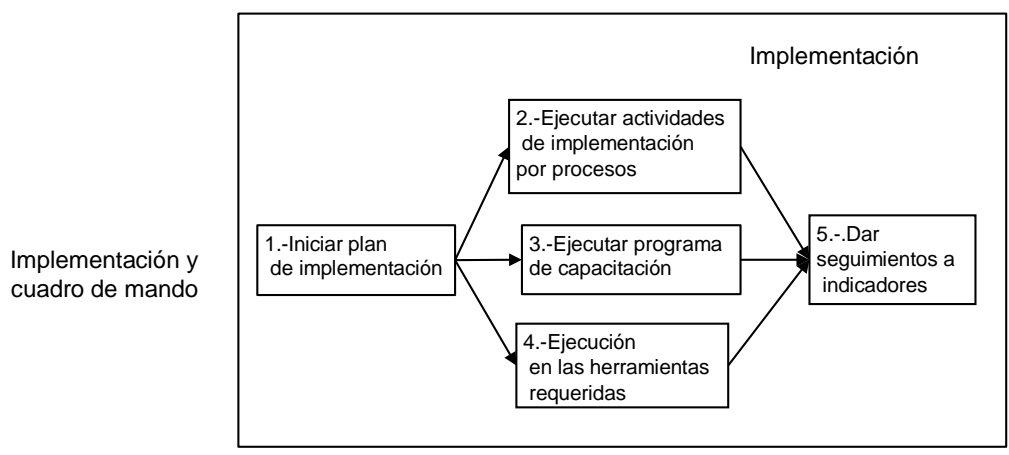

Figura 6 - Cuarta relación (Ocampo 2009) 


\section{Quinta relación}

Aquí se da por terminado la automati ación, teniendo en ejecución la monitorización en ti real permiti el rediseño de los procesos, auditoría de los procesos, análisis de indicadores y como consecuencia la mejora conti

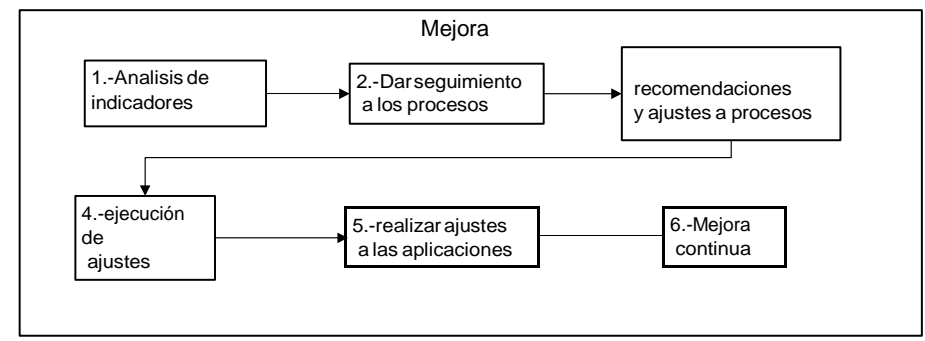

Cuadro de mando

Figura 7 - Quinta relación (Ocampo 2009)

\section{Conclusiones}

La relación de implementación entre el nivel de madurez BPM y el proceso de gestión de servicios, permiten poner en práctica el enfoque de procesos y las buenas prácticas propuestas por ITIL, añadiendo continuidad a la mejora de procesos y por consiguiente mejora a los servicios de TI.

El gobierno de TI será eficiente a medida que lo sean los procesos que propone ITIL.

\section{Recomendaciones}

La orientación de este artí aún no es aplicada a una ejecución real, quedando plasmada solo en la parte teórica de la misma, se recomienda poder llevarlo a la prácti a para poder sacar conclusiones de carácter experimental y cuantifi able.

Joseph Cruz

Universidad Peruana Unión e-mail: josephibrahim@operamail.com

Daniel Lévano

Universidad Peruana Unión e-mail: fia.tarapoto@upeu.edu.pe

Recibido: 20 de octubre de 2011 Aceptado: 08 de noviembre de 2011 


\section{Referencias}

Carrasco, L. (2006). May. Implantación de herramientas de BPM. Un enfoque novedoso, realista e independiente. [Resumen en línea]. Fornmato ppt. Disponibilidad libre en: <http:// www.delphinph.com>.

Garimella, K., Lees. M., Williams, B. (s/f). Introducción a BPM para Dummies [Libro en línea]. [Consultado el 20 de julio de 2011]. Documento en pdf. Disponibilidad libre en: <http:// www.sotfwareag.com/latam/res/books/bpm_for_dummies/default.asp>.

Ocampo, C. (2009), May. Implementación de modelo de procesos de gestión de servicios con ITIL (Information Technology Infrastructure Library). [Resumen en línea]. Formato pdf. Disponibilidad libre en: <http:// www.utp.edu.co>

TCP Sistemas e Ingeniería. (2011). Gestión de procesos de negocio. 1.0. Brazil. TCPsi. [Consultado el 15 de agosto del 2011] Disponible libre en:< www.tcpsi.com/ >.

Vásquez, G., González, L., León, R. (2010). Proceso de implementación de una plataforma BPM (Business Process Management) [artículo en línea]. [Consultado el 25 de julio de 2011]. Documento en pdf. Disponibilidad libre en: <http://www.eatis.org/eatiz2010/portal/paper/memoria/html/files/95.pdf >. 